\title{
Reliability of a tool for assessing mobility in wheelchair-dependent paraplegics
}

\author{
Lisa A Harvey ${ }^{1}$, Julia Batty ${ }^{1}$ and Alex Fahey ${ }^{2}$ \\ ${ }^{1}$ Department of Physiotherapy, The Prince Henry Hospital, Sydney, Australia; ${ }^{2}$ Fitability, The University of Sydney, \\ Sydney, Australia
}

\begin{abstract}
The aim of this study was to design and test the reliability of an assessment tool that could be used by physiotherapists to quantify the mobility of wheelchair-dependent paraplegics. The assessment tool examined six key tasks fundamental to the mobility of wheelchair-dependent paraplegics, comprising moving from lying to sitting, completing a horizontal transfer, completing a vertical transfer, pushing on flat ground, pushing on ramps and negotiating kerbs. A six-point scoring system was used to assess the level of mobility attained by subjects on each task. The scoring system was different for each task and took into account the level of assistance and time required to complete the task or the complexity of the task. Twenty wheelchair-dependent paraplegics were assessed independently by two therapists using the assessment tool. The Cohen's Weighted Kappas describing inter-rater reliability of each task ranged between $0.82-0.96$, indicating a high reliability between therapists. It was concluded that the assessment tool is a reliable way of assessing the mobility of wheelchair-dependent paraplegics.
\end{abstract}

Keywords: paraplegia; independence; mobility; physical therapy; assessment

\section{Introduction}

The primary aim of physiotherapy for wheelchairdependent paraplegics is to optimise mobility. Presently, however, there is no sensitive tool for measuring mobility which is suitable for use with this population. Such a measurement tool would enable physiotherapists to quantify the effects of therapy, and in turn to conduct research, justify funding and communicate effectively amongst health professionals.

There are many measures used to quantify a person's level of disability. Some measures such as the Functional Independent Measure (FIM), ${ }^{1}$ the Barthel Index ${ }^{2}$ and the Modified Barthel Index, ${ }^{3,4}$ were designed to be used with many different types of disabled populations. Others, like the Spinal Cord Independent Measure (SCIM) ${ }^{5}$ and The Quadriplegic Index of Function ${ }^{6,7}$ were specifically intended for the spinal cord injured population. None, however, were specifically designed to assess the mobility of paraplegics. Whilst these measures are useful for certain purposes, they do not distinguish important functional differences in the mobility of wheelchairdependent paraplegics.

The two scales most commonly used for the spinal cord injured population are the Modified Barthel Index ${ }^{3,4,7}$ and the FIM. ${ }^{7-9}$ Yet neither of these scales

Correspondence: L Harvey, School of Physiotherapy, Faculty of Health Sciences, University of Sydney, P.O. Box 170, Lidcombe, NSW, Australia, 2141 distinguish between levels of mobility commonly seen in paraplegics. For example, with both scales, all patients capable of pushing a wheelchair outside receive the same score, regardless of their competency at this skill. Likewise, a patient capable of transferring from a wheelchair to a bed receives the same score as a patient capable of far more complex and difficult skills, such as transferring from floor to wheelchair.

The poor sensitivity of the FIM has recently been demonstrated in a study of 100 spinal injured patients. ${ }^{10}$ This study found no difference in the group FIM scores of $\mathrm{C}_{8}$ quadriplegics. $\mathrm{T}_{1-5}$ paraplegics and $\mathrm{T}_{5-12}$ paraplegics. An earlier study ${ }^{9}$ found little variation in the FIM scores of high $\left(\mathrm{T}_{3-4}\right)$ and low $\left(T_{11}\right)$ level paraplegics or of young $(<30$ years) and older ( $>45$ years $)$ paraplegics, yet clinical observations would suggest that the mobility of these groups of patients would differ substantially.

An additional and related problem with the available scales is that they impose a ceiling effect; that is, they do not assess patients' abilities to perform advanced mobility skills such as ascending kerbs and getting on and off the floor. This is despite the fact that competency in these advanced skills can make a significant difference to quality of life. The ceiling effect of the FIM may explain why Marino et al ${ }^{8}$ found no improvement in patients in the 3 month period after injury, and why Yarkony et $a l^{3}$ found that patients with spinal cord injuries do not continue to improve in the 3 year period following discharge. 
Despite this insensitivity and ceiling effect of the available measures, they are increasingly being used by funding bodies to monitor the success of rehabilitation programs for paraplegics. This may be inappropriate because the effectiveness of therapeutic interventions are not being adequately measured in these scales. There is therefore a need for a sensitive yet simple assessment scale that can be routinely used to determine the mobility of wheelchair-dependent paraplegics. The aim of this study was to design and test the reliability of an assessment tool that could be used to quantify the mobility of wheelchair dependent paraplegics.

\section{Methods}

\section{The assessment tool}

Over a 2 year period a group of physiotherapists experienced in the physiotherapy management of individuals with paraplegia devised and trialed the assessment tool. Initially, the tool quantified patients' abilities to perform an extensive range of the mobility skills typically learnt in physiotherapy programs. However, whilst the resultant assessment tool was comprehensive, it was complicated and took an excessive amount of time to administer. The assessment tool was further modified and the final version included just six key mobility tasks. The tasks selected included simple and complex skills and were representative of the array of motor tasks learnt by patients. They included moving from lying to sitting, completing a horizontal transfer, completing a vertical transfer, pushing a wheelchair on the flat, pushing a wheelchair up and down ramps, and negotiating kerbs in a wheelchair. For each task, subjects were scored on a six-point scale, with 6 representing the best score and 1 the worst. The scoring system for each task was based on a logical progression taking into account the level of assistance required, the time taken to complete the task and the complexity of the task. It was not intended that the scores for each task be summed to give an overall score.

\section{Testing for inter-rater reliability}

Twenty wheelchair dependent paraplegics volunteered from a group of in-patients and out-patients to participate in the study. All had lesions between $T_{2}$ and $\mathrm{L}_{2}$ and used a wheelchair as their primary mode of locomotion.

Subjects were independently assessed by two physiotherapists experienced in the management of spinal cord injured patients. Each subject performed the six tasks in the same order but the order in which the therapists tested subjects was randomised. Each subject performed both assessments on the same day with a short break between the two.

The scores were analyzed for their inter-rater reliability with Cohen's Weighted Kappa. ${ }^{11}$ This statistic measures the degree of agreement between two scores above that which would occur by chance. The weighting used to describe the experimenters' perceptions of the severity of different degrees of disagreement was decided prior to the study. A difference of one point was denoted a weighting of 0.9 , whilst a difference of five points was denoted a weighting of 0.0 . Difference of two, three and four points were weighted by $0.8,0.4$ and 0.2 , respectively. In addition, after data from the six different tasks were pooled, percent close agreements between the two therapists' scores were calculated.

\section{Results}

The mean $( \pm$ SD) age was 45.6 years $( \pm 16.8)$ and the median (and inter-quartile range) time since injury was 9 years $(2.5-18)$. Levels of mobility attained on each of the six tasks is given in Table 1. These values were obtained after taking the mean of the two assessors' scores for each subject. The Weighted Kappa for each task ranged between $0.82-0.96$, reflecting a strong agreement between the two therapists (see Table 1). The proportion of chance agreement $(\mathrm{Pc})$ and observed agreement (Po) for each task is also detailed in Table 1.

When the data from the six tasks were pooled, the two therapists' scores were in perfect agreement $82 \%$ of the time, and were within one grade of each other $17 \%$ of the time. Their scores only differed by two grades $2 \%$ of the time and never differed by three or more grades.

\section{Discussion}

Whilst physiotherapists are generally aware of the need to objectively measure the effects of their interventions, they do not have a standardised, accepted or reliable method of assessment for wheelchair-dependent paraplegics. Consequently, there is no uniform approach to the assessment of these patients, and physiotherapists have increasingly been relying on gross measures of disability such as the Modified Barthel Index, ${ }^{3,4}$ the Functional Independence Measure ${ }^{1}$ and the Quadriple-

Table 1 The median (and inter-quartile range) levels of mobility attained on each of the six tasks

\begin{tabular}{lcccc}
\hline Task no. & Median & $\begin{array}{c}\text { Weighted } \\
\text { Kappa }\end{array}$ & Po & $P c$ \\
\hline 1 & $6(3.75-6)$ & 0.94 & 0.985 & 0.748 \\
2 & $6(6-6)$ & 0.82 & 0.985 & 0.918 \\
3 & $3.5(1-6)$ & 0.98 & 0.990 & 0.537 \\
4 & $5(4.5-5.25)$ & 0.94 & 0.990 & 0.828 \\
5 & $5(4.25-5.0)$ & 0.89 & 0.980 & 0.820 \\
6 & $3.5(2-5.25)$ & 0.83 & 0.950 & 0.708 \\
\hline
\end{tabular}

The median value was obtained from the mean of the two assessors' scores for each subject. The weighted Kappa, confidence interval, proportion of observed agreement (Po) and proportion of chance agreement $(\mathrm{Pc})$ for each task are also shown 
gic Index of Function. $^{6}$ However, none of these alternatives are sensitive scales appropriate for measuring the effects of physiotherapy interventions in wheelchair-dependent paraplegics.

The assessment scale described here is simple and meaningful, and includes a diversity of the tasks fundamental to the mobility of wheelchair-dependent paraplegics. This scale has validity because the scoring system is based on a logical progression and the skills tested are necessary for functional mobility. It can be completed by a physiotherapist in under $15 \mathrm{~min}$ and requires no special equipment. The results quickly summarise the level of mobility of patients in a manner that is readily understood by other professionals.

Some of the tasks included in the assessment tool are simple, such as getting from lying to sitting, whilst others are more complex, such as changing a kerb and moving from the floor to the wheelchair. It was therefore not surprising to find that with the simpler task (ie, task number 2), subjects obtained higher scores, and with more complex tasks (ie, tasks number 3 and 6) subjects obtained lower scores (see Table 1). The inclusion of tasks of differing complexities make the tool applicable to measuring the mobility of competent and less competent patients. However, this also makes it more difficult to meaningfully add the scores from each task into a grand total. We therefore recommend that the scores should be interpreted in relation to the task to which they refer.

In conclusion, this study has described a simple and reliable assessment tool that can be used by physiotherapists to assess and quantify the mobility of wheelchair-dependent paraplegics and can therefore be used to quantify the effects of therapeutic interventions.

\section{Acknowledgements}

The authors acknowledge the contribution of Lynne Blanche, Jenni Barron, and Jayne Callangham in the design of the assessment tool. They also acknowledge the statistical advice given to them by Chris Maher and Robert Herbert.

\section{References}

1 Data management service (of the uniform data system for medical rehabilitation and the center for uniform assessment research): Guide for use of the uniform data set for medical rehabilitation. ver. 3.0. The Buffalo general hospital/State University of New York at Buffalo, 1990.

2 Granger CV, Albrecht GL, Mamilton BB. Outcomes of comprehensive medical rehabilitation: measurement by PULSES Profile and the Barthel Index. Arch Phys Med Rehabil 1979; 60: $145-154$.

3 Yarkony GM et al. Functional skills after spinal cord injury rehabilitation: three-year longitudinal follow-up. Arch Phys Med Rehabil 1988; 69: $111-114$.

4 Yarkony GM, Roth EJ, Heinemann AW, Lovell L. Rehabilitation outcomes in C6 tetraplegia. Paraplegia 1988; 26: 177-185.

5 Catz A. A new scale for measuring disability in patients with paraplegia and tetraplegia. (Abstract) Proceedings of the 35th Annual Scientific Meeting of International Medical Society of Paraplegia, Atlanta, Georgia, USA 1996.

6 Gresham G et al. The Quadriplegia Index of Function (QIF): Sensitivity and reliability demonstrated in a study of thirty quadriplegic patients. Paraplegia 1986; 24: 38 - 44.

7 Marino R, Stineman M. Functional assessment in spinal cord injury. Top Spinal Cord Inj Rehabil 1996; 1: $32-45$.

8 Marino RJ et al. Assessing selfcare status in quadriplegia: comparison of the quadriplegia index of function (QIF) and the functional independence measure (FIM). Paraplegia 1993; 31: $225-233$.

9 Menter MM et al. Impairment, disability, handicap and medical expenses of persons aging with spinal cord injury. Paraplegia 1991; 29: $613-619$.

10 Ota $\mathrm{T}$ et al. Functional assessment of patients with spinal cord injury: measured by the motor score and the Functional Independence Measure. Spinal Cord 1996; 34: 531 - 535.

11 Haas M. Statistical methodology for reliability studies. J Manip Phys Ther 1991; 14: $119-132$.

\section{Appendix: Assessment Tool}

\section{General}

Patients are assessed on their ability to perform six different tasks. For each task patients are scored on a six-point scale, with 6 representing the best score and 1 the worst. Patients must perform the tasks in the same order, as set out below. With tasks number 2 and 6 , the patient may have three attempts with the best score recorded. The scores for each task were not designed to be added or combined in any way. If a test is not attempted, 'not applicable' should be recorded, not a score of 1 . For the tasks where assistance is quantified the following definitions apply:

Total assistance. A situation where the patient provides less than $25 \%$ of the effort required to complete the task and the clinician provides the remaining effort.

Maximal assistance. A situation where the patient provides between $25 \%$ and $49 \%$ of the effort required to complete the task and the clinician provides the remaining effort.

Moderate assistance. A situation where the patient provides between $50 \%$ and $74 \%$ of the effort required to complete the task and the clinician provides the remaining effort.

Minimal assistance. A situation where the patient provides between $75 \%$ and $99 \%$ of the effort required to complete the task and the clinician provides the remaining effort.

Supervision. A situation where the patient provides $100 \%$ of the effort to complete the task and the clinician provides either verbal prompts or supervision. However, the clinician does not provide any physical assistance.

Independent. A situation where the patient provides $100 \%$ of the effort required to complete the 
task and the patient does not require any supervision or verbal prompts.

\section{Task 1: Supine to long sitting}

This task involves the patient moving from supine to long sitting on a wide plinth. The patient must not hold on to the side of the plinth or any external aids (eg trapeze).

A score of 1 indicates that the patient completes the task with total assistance.

A score of 2 indicates that the patient completes the task with maximal assistance.

A score of 3 indicates that the patient completes the task with moderate assistance.

A score of 4 indicates that the patient completes the task with minimal assistance.

A score of 5 indicates that the patient completes the task with supervision.

A score of 6 indicates that the patient completes the task independently.

\section{Task 2: Horizontal transfer}

This task involves the patient transferring from their normal wheelchair to a plinth that is the same height as the top of their seat cushion. The front corner of the wheelchair must be $20 \mathrm{~cm}$ from the plinth. Subjects may use a slide board, but this will lower their score by one point.

A score of 1 indicates that the patient completes the transfer with total assistance.

A score of 2 indicates that the patient completes the transfer with maximal assistance.

A score of 3 indicates that the patient completes the transfer with moderate assistance.

A score of 4 indicates that the patient completes the transfer with minimal assistance.

A score of 5 indicates that the patient completes the transfer with supervision.

A score of 6 indicates that the patient completes the transfer independently.

\section{Task 3: Vertical transfer}

This task involves the patient transferring from the floor back into their wheelchair. To complete the task independently the patient must be sitting on their cushion in their wheelchair. The wheelchair may be put on the plinth, if the patient does not wish to transfer onto the floor.

A score of 1 indicates that the patient completes the transfer with total assistance.
A score of 2 indicates that the patient completes the transfer with maximal assistance.

A score of 3 indicates that the patient completes the transfer with moderate assistance.

A score of 4 indicates that the patient completes the transfer with minimal assistance.

A score of 5 indicates that the patient completes the transfer with supervision.

A score of 6 indicates that the patient completes the transfer independently.

\section{Task 4: Push on flat}

This task involves the patients pushing their wheelchair on level ground. Two marks (or objects eg witches hats) must be placed $25 \mathrm{~m}$ apart on level ground. The task requires the patient to push from one mark, around the second mark and back to the original mark. Timing starts and finishes when the front wheel moves past the first mark. One circuit is defined as pushing 50 metres, ie pushing from the first marker to the second marker and back to the first.

A score of 1 indicates that the patient cannot independently push $25 \mathrm{~m}$ (ie, 0.5 of a circuit) in less than $1 \mathrm{~min}$.

A score of 2 indicates that the patient independently pushes $25 \mathrm{~m}$ (ie, 0.5 of a circuit) in less than $1 \mathrm{~min}$, but cannot independently push $50 \mathrm{~m}$ in less than $1 \mathrm{~min}$.

A score of 3 indicates that the patient independently pushes $50 \mathrm{~m}$ (ie, 1 circuit) in less than $1 \mathrm{~min}$, but cannot independently push $200 \mathrm{~m}$ (ie, 4 circuits) in less than $4 \mathrm{~min}$.

A score of 4 indicates that the patient independently pushes $200 \mathrm{~m}$ (ie, 4 circuits) in less than $4 \mathrm{~min}$, but cannot independently push $200 \mathrm{~m}$ (ie, 4 circuits) in less than $3 \mathrm{~min}$.

A score of 5 indicates that the patient independently pushes $200 \mathrm{~m}$ (ie, 4 circuits) in less than $3 \mathrm{~min}$, but cannot independently push $200 \mathrm{~m}$ (ie, 4 circuits) in less than $1.5 \mathrm{~min}$.

A score of 6 indicates that the patient independently pushes $200 \mathrm{~m}$ (ie, 4 circuits) in less than $1.5 \mathrm{~min}$.

\section{Task 5: Push on ramp}

This task involves the patient pushing their wheelchair on a 1:12 ramp. Two marks (or objects, eg witches hats) must be placed on the ramp 15 metres apart. The task requires the patient to push from the bottom mark, up the ramp, around the second mark and back down to the original mark. Timing starts and finishes when the front wheel moves past the mark. One circuit is defined as pushing 30 metres, ie, from the first marker, up to the second marker and back down to the first. 
A score of 1 indicates that the patient cannot independently push up or down the ramp.

A score of 2 indicates that the patient independently pushes either up or down the ramp but not both.

A score of 3 indicates that the patient independently pushes up and down the ramp (ie, completes 1 circuit) but takes more than 2 min.

A score of 4 indicates that the patient independently pushes up and down the ramp (ie, completes 1 circuit) in less than $2 \mathrm{~min}$, but not in less than $1 \mathrm{~min}$.

A score of 5 indicates that the patient independently pushes up and down the ramp (ie, completes 1 circuit) in less than $1 \mathrm{~min}$, but not in less than $15 \mathrm{~s}$.

A score of 6 indicates that the patient independently pushes up and down the ramp (ie, completes 1 circuit) in less than $15 \mathrm{~s}$.

\section{Task 6: Negotiate kerbs}

This task involves the patient pushing up kerbs in their wheelchair. A small kerb is defined as one that is approximately $2.5 \mathrm{~cm}$ high and a large kerb is defined as one that is $15 \mathrm{~cm}$ high. The task requires the patient to start below the kerb, push up the kerb and finish above the kerb.

A score of 1 indicates that the patient cannot independently push up a small kerb.

A score of 2 indicates that the patient independently pushes up a small kerb, but cannot achieve score 3 .

A score of 3 indicates that the patient pushes up a large kerb with a pole and minimal assistance.

A score of 4 indicates that the patient pushes up a large kerb with minimal assistance.

A score of 5 indicates that the patient pushes up a large kerb with supervision.

A score of 6 indicates that the patient independently pushes up a large kerb. 